\title{
Serum Levels of S-Glutathionylated Proteins as a Risk-Marker for Arteriosclerosis Obliterans
}

\author{
Kazuki Nonaka, MD; Noriaki Kume, MD ${ }^{*}$; Yoshishige Urata, PhD; \\ Shinji Seto, MD*; Takaaki Kohno, BS*; Sumihisa Honda, PhD*; \\ Soji Ikeda, MD*; Takahiro Muroya, MD**; Yosihiko Ikeda, MD ${ }^{\dagger}$; \\ Yoshito Ihara, MD; Toru Kita, MD†; Takahito Kondo, MD
}

\begin{abstract}
Background Oxidative stress plays a role in the development of chronic peripheral arterial disease (PAD) because under these conditions redox regulation is impaired, inducing the S-glutathionylation of proteins. A method of estimating the levels of S-glutathionylated proteins has been developed using biotinylated glutathione S-transferase, which allows the study of their crucial role in the oxidative stress-related progression of PAD.

Methods and Results The serum levels of S-glutathionylated proteins were examined in 41 patients with arteriosclerosis obliterans (ASO) and 38 age-matched non-ASO patients using biotinylated glutathione S-transferase. The levels were higher in the patients with ASO, even early on, and positively correlated with the ankle/brachial index. In vitro, the levels of S-glutathionylated proteins were reduced in the presence of glutathione and glutaredoxin.
\end{abstract}

Conclusions Serum levels of S-glutathionylated proteins are a sensitive risk-marker for ASO at an early stage. (Circ J 2007; 71: 100-105)

Key Words: Arteriosclerosis obliterans; Oxidative stress; S-glutathionylation

$\mathbf{T}$ he number of patients suffering from arteriosclerosis obliterans (ASO) is anticipated to increase, accompanying the increase in incidence of risk factors such as obesity, hypercholesteremia, diabetes, and hypertension! Pathologically, ASO derives from atherosclerosis, and complete occlusions by fresh or old thrombi are often observed. Treatment includes anticoagulants, antiplatelet drugs, and vasodilators, and in the advanced stages pericutaneous transluminal angioplasty, bypass surgery, and prosthetic arterial grafts have been used. New approaches include intravenous administration of prostaglandin $\mathrm{E}_{1}^{2}$ or gene therapy with hepatocyte growth factor; both aimed at increasing peripheral blood flow. Most patients with ASO have no apparent clinical symptoms early on, but diagnosis at the early stage is essential for preventing progression. Unfortunately, there are currently no specific and sensitive markers for ASO, so the aim of this study was to find a new risk-marker for the diagnosis of ASO in the earlier stages.

The development of atherosclerosis is induced by severe damage to endothelial cells from various pro-inflammatory cytokines, adhesion molecules, or sheer stress, for example ${ }^{4-6}$ Furthermore, oxidative stress is believed to play a

(Received June 5, 2006; revised manuscript received October 6, 2006; accepted October 13, 2006)

Department of Biochemistry and Molecular Biology in Disease, *Department of Radiation Epidemiology, Atomic Bomb Disease Institute, **Department of Internal Medicine, Nagasaki University Graduate School of Biomedical Sciences, Nagasaki, ${ }^{\dagger T a g a w a}$ Municipal Hospital, Tagawa and Department of Cardiovascular Medicine, Graduate School of Medicine, Kyoto University, Kyoto, Japan

Mailing address: Takahito Kondo, MD, Department of Biochemistry and Molecular Biology in Disease, Atomic Bomb Disease Institute, Nagasaki University Graduate School of Biomedical Sciences, 1-12-4 Sakamoto, Nagasaki 852-8523, Japan. E-mail: kondo@net.nagasaki-u. ac.jp crucial role in the progression of peripheral arterial disease (PAD), because it induces modifications of cellular components such as proteins, lipids, and DNA, leading to cell dysfunction or apoptosis. Most of the risk factors for PAD, such as smoking, obesity, hypertension, diabetes, and hypercholesterolemia, create oxidants that damage endothelial cells? The cysteine thiols of proteins are easily modified by oxidative stress when the antioxidative systems are suppressed and under oxidative stress caused by reactive oxygen species or nitrogen oxide species, it is the sulfhydryl residues of proteins that are most susceptible. In response, the sulfhydryl groups are oxidized to form disulfides in a reaction with the reduced form of glutathione disulfide (GSSG) or converted irreversibly to sulfenic, sulfinic, and sulfonic acid derivatives!1 S-Glutathionylated proteins reported to date include glyceraldehyde-3-phosphate dehydrogenase ${ }^{12}$ annexin $\mathrm{A} 2{ }_{2}^{13}$ protein kinase $\mathrm{C}^{14}$ and carbonic anhydrase III! $!^{15}$ The S-glutathionylation of proteins is initiated in the presence of GSSG? The S-glutathionylation of the sulfhydryl groups changes a protein's function, and the process is regulated by thioredoxin (TRX) or glutathione (GSH)/glutaredoxin (GRX). Such modifications of proteinthiols by oxidative stress are speculated to occur in patients with PAD; however, no data on changes in the levels of serum S-glutathionylated proteins have been reported for patients with peripheral or cardiovascular diseases such as stroke, coronary artery disease, and end-stage renal disease. We are interested in the S-glutathionylation of proteins in ASO patients and so the aim of the present study was to evaluate the serum levels in these patients, as a risk-marker for ASO in the early stages, because elevation supposedly reflects redox imbalance. 
Table 1 Characteristics of Patients With ASO

\begin{tabular}{|c|c|c|c|c|}
\hline & \multirow{2}{*}{ Non-ASO patients } & \multicolumn{3}{|c|}{ ASO patients (Fontaine) } \\
\hline & & Stage 1 & Stage 2 & Stage 3 \\
\hline Patients, $n$ & 38 & 9 & 22 & 10 \\
\hline$A B I($ mean $\pm S D)$ & $0.94 \pm 0.02$ & $0.72 \pm 0.07$ & $0.41 \pm 0.13$ & $0.21 \pm 0.06$ \\
\hline Age $($ mean $\pm S D)$, years & $66 \pm 11$ & $73 \pm 8$ & $72 \pm 7$ & $71 \pm 5$ \\
\hline Male sex, $n(\%)$ & $15(39)$ & $6(67)$ & $16(73)$ & $8(80)$ \\
\hline \multicolumn{5}{|l|}{ Risk factors, $n(\%)$} \\
\hline Hypertension & $17(45)$ & $7(78)$ & $19(86)^{\dagger}$ & $9(90)^{\dagger}$ \\
\hline Diabetes & $23(61)$ & $3(33)$ & $6(27)^{+}$ & $3(30)$ \\
\hline Smoking & $9(24)$ & $6(67)^{\S}$ & $15(68)^{\S}$ & $5(50)$ \\
\hline Hypercholesterolemia & $16(42)$ & $4(44)$ & $9(41)$ & $5(50)$ \\
\hline Chronic renal failure on hemodialysis & 0 & 0 & 0 & 0 \\
\hline Angina & $4(11)$ & $2(22)$ & $4(18)$ & $3(30)$ \\
\hline \multicolumn{5}{|l|}{ Lipid profile (mean $\pm S D)$} \\
\hline Total cholesterol, $\mathrm{mg} / \mathrm{dl}$ & $253 \pm 45$ & $198 \pm 23 *$ & $205 \pm 28 *$ & $202 \pm 17 *$ \\
\hline$L D L-C, m g / d l$ & $153 \pm 46$ & $111 \pm 24 \pi$ & $119 \pm 23 \pi$ & $116 \pm 14 \pi$ \\
\hline$h s-C R P($ mean $\pm S D), n g / m l$ & $2.19 \pm 0.42$ & $3.17 \pm 1.01$ & $3.57 \pm 0.85^{\#}$ & $4.30 \pm 0.99^{\#}$ \\
\hline
\end{tabular}

Values for hs-CRP were transformed in logarithm of 10. One-way ANOVA was followed up with Tukey-Kramer pairwise comparisons among means.

${ }^{\dagger} p<0.05$ for comparison with non-ASO patients; ${ }^{+} p<0.05$ for comparison with non-ASO patients; ${ }^{\S} p<0.05$ for comparison with non-ASO patients; * $p<0.005$ for comparison with non-ASO patients; ${ }^{q} p<0.05$ for comparison with non-ASO patients; ${ }^{*} p<0.05$ for comparison with non-ASO patients.

ASO, arteriosclerosis obliterans; ABI, ankle/brachial index; $L D L-C$, low-density lipoprotein-cholesterol; hs-CRP, high-sensitivity C-reactive protein.

\section{Methods}

\section{Patient Sample}

We enrolled 41 patients diagnosed with ASO. All of them had the characteristic complaints of chronic limb ischemia, including intermittent claudication, rest pain, or non-healing ischemic ulcers (Fontaine I, $\mathrm{n}=9$; Fontaine II, $\mathrm{n}=22$; Fontaine III, $\mathrm{n}=10$ ) as confirmed by angiography. Of the patients visiting hospital without apparent PAD, we recruited 38 age-matched controls. All participants gave written informed consent and prior to the commencement of the present study, the protocol was approved by the ethics committees of all the participating universities and hospitals.

\section{Measurement of Ankle/Brachial Index (ABI)}

Blood pressure, heart rate, and $\mathrm{ABI}$ were measured using the Form pulse wave velocity (PWV)/ABI non-invasive vascular screening device (Nihon Colin Inc, Tokyo, Japan) after the subject had rested supine for at least $20 \mathrm{~min}$. ABI was calculated 2 or 3 times for both legs and averaged; an $\mathrm{ABI}<0.9$ was considered to indicate the presence of disease.

\section{Immunoblot Analysis}

Unless otherwise indicated, $20 \mu \mathrm{g}$ samples of serum was used. Protein concentrations were determined using a BCA assay kit (Pierce, Rockford, IL, USA). Samples were electrophoresed on 5\% sodium dodecyl sulfate-polyacrylamide gels (SDS-PAGE) in the absence of dithiothreitol (DTT) and the proteins in the gels were transferred onto nitrocellulose membranes. The membranes were blocked in Tris-buffered saline $(10 \mathrm{mmol} / \mathrm{L}$ Tris- $\mathrm{HCl}[\mathrm{pH} 7.5]$ and $0.15 \mathrm{~mol} / \mathrm{L} \mathrm{NaCl}$; TBS) containing $0.05 \%$ Tween 20, v/v (TBST), and 5\% (w/v) nonfat dry milk, then reacted with primary antibodies in TBST containing 3\% (w/v) bovine serum albumin overnight with constant agitation at $4^{\circ} \mathrm{C}$. After several washes with TBST, the membranes were incubated with horseradish peroxidase (HRP)-conjugated anti-IgG antibodies. Proteins in the membranes were then visualized using an enhanced chemiluminescence detection kit (Amersham Biosciences) according to the manufacturer's instructions. Levels of highsensitivity C-reactive protein (hs-CRP) were determined in the same serum samples used for SLOX-1, with a commercially available electrochemiluminescent immunoassay kit (F. Hoffman-La Roche Ltd).

\section{Detection of S-Glutathionylated Proteins by Biotin- Glutathione S-Transferase (GST) on Blotted Membranes}

Serum levels of S-glutathionylated proteins were estimated according to the methods described by Cheng et $\mathrm{al}^{8}$ using biotinylated GST. Serum samples were collected serially and stored at $-80^{\circ} \mathrm{C}$ until assays were performed. Of each sample, $20 \mu \mathrm{g} / \mathrm{lane}$ were subjected to $5 \%$ SDS-PAGE under non-reducing conditions. The proteins in the gels were transferred onto nitrocellulose membranes, which were blocked in phosphate bufferd salin (PBS) containing $0.1 \%$ Tween $20, \mathrm{v} / \mathrm{v}$, and $5 \%$ nonfat dry milk, then treated with BSA containing 5\% (w/v) bovine serum albumin for $2 \mathrm{~h}$ at room temperature and further incubated with $30 \mathrm{mg} / \mathrm{ml}$ biotin-GST overnight. After several washes with PBS, the membranes were incubated with HRP-conjugated streptavidin (1:1,000 dilution) for $1 \mathrm{~h}$ at room temperature. Peroxidase activity was detected after treatment with $2 \mathrm{mmol} / \mathrm{L}$ hydrogen peroxide and $0.6 \mathrm{mg} / \mathrm{ml}$ 4-chloro-1-naphthol in PBS.

\section{Statistical Analysis}

Statistical analysis was performed using Stat-View (version 4.5, Abacus Concepts Inc, Calabasus, CA, USA) and R. The 1-way ANOVA was used to compare continuous variables, with the Tukey-Kramer test for multiple comparisons, and 2-way cross-tabulation with the chi-square test was used for binary variables, when appropriate, to compare differences between groups. Statistically significant differences among groups were analyzed by the Kruskal-Wallis test with Dunn's test. When S-glutathionylated proteins 
A

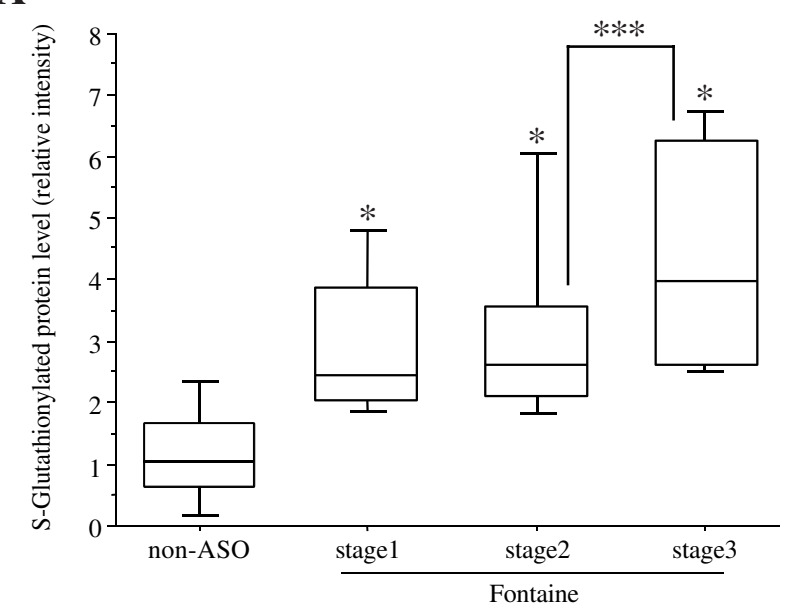

B

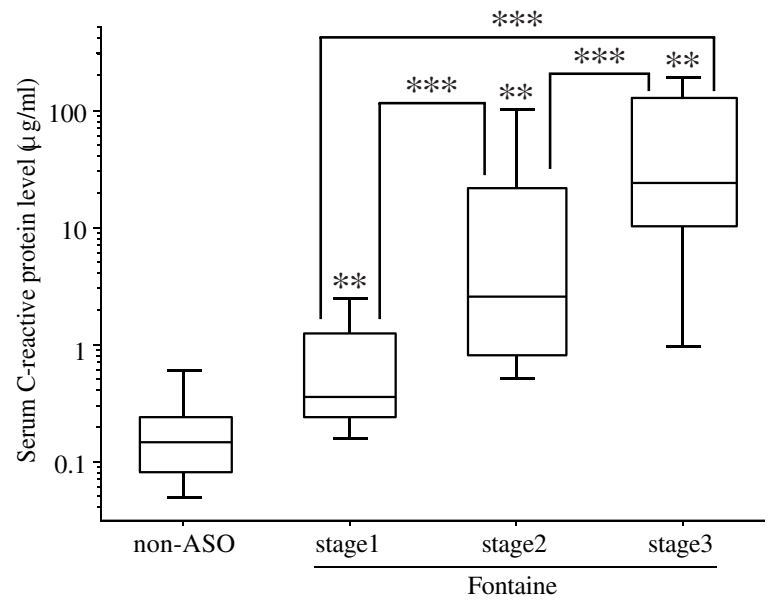

Fig 1. Serum levels of S-glutathionylated proteins and high-sensitivity $\mathrm{C}$-reactive protein (hs-CRP). S-glutathionylated protein levels are expressed as relative intensity compared with non-ASO patients (A), and hs-CRP $\mu \mathrm{g} / \mathrm{ml}$ ) levels (B) were determined in 41 ASO patients (9 at Fontaine stage 1, 22 at stage 2, and 10 at stage 3) and 38 non-ASO patients. Data are indicated in box plots. Center horizontal lines indicate median values; upper and lower edges of outer boxes, $25^{\text {th }}$ and $75^{\text {th }}$ percentiles; and lower and upper bars, $10^{\text {th }}$ and $90^{\text {th }}$ percentiles. Statistically significant differences among the 4 groups by KruskalWallis test with Dunn's test. *p $<0.0001$ vs non-ASO patients; **p $<0.05$ vs non-ASO patients; $* * * \mathrm{p}<0.05$. ASO, arteriosclerosis obliterans.

were undetectable by immunoblot analysis, a score of 0 was assigned. Any association between S-glutathionylated proteins and hs-CRP, total cholesterol or low-density lipoprotein (LDL)-cholesterol (C) was evaluated with Spearman's rank correlation coefficient. Logarithmic values of hs-CRP were used as variables for statistical analyses. The effect of S-glutathionylated proteins on ABI was analyzed by using a multiple linear regression model with hyperlipidemia, hypertension, smoking, diabetes mellitus, and hs-CRP as covariates. The squared multiple correlation coefficient $\left(\mathrm{R}^{2}\right)$ was calculated as a goodness-of-fit measure. Values of $\mathrm{p}<0.05$ were considered statistically significant.

\section{Results}

\section{Clinical Characteristics of the Study Group}

Table 1 summarizes age, gender, conventional vascular risk factors, ABI, lipid profile, and levels of hs-CRP. Pa- tient characteristics, including age and the incidence of hypercholesterolemia and angina, were comparable between the ASO and non-ASO groups. The ratio of males to females was higher in the ASO groups. Of the risk factors, the rate of hypertension was higher at Fontaine stages 2 and 3 in the ASO groups than in the non-ASO group $(\mathrm{p}<0.05)$, and the rate of smoking was higher at Fontaine stages 1 and 2 in the ASO groups $(\mathrm{p}<0.05)$. There was no difference in the rate of angina between the ASO and non-ASO groups. In this study, patients with chronic renal failure on hemodialysis were excluded. The serum concentrations of total cholesterol and LDL-C were lower in the ASO groups than in the non-ASO group $(\mathrm{p}<0.05)$.

\section{Serum Levels of S-Glutathionylated Protein}

Fig 1A shows the estimated S-glutathionylated protein levels in serum samples from the ASO groups. Statistically significant differences were found among the 4 groups (Kruskal-Wallis test). The median level of S-glutathionylated proteins was 1.06 in non-ASO patients, 2.46 at stage $1,2.62$ at stage 2 , and 3.97 at stage 3 . The number of males in the non-ASO group was less than in the ASO groups; however, in a preliminary study, there was no difference in the levels of S-glutathionylated proteins between the sexes (data not shown). The levels were increased at every stage of ASO compared with the non-ASO patients $(\mathrm{p}<0.0001)$. A significant difference in the levels of S-glutathionylated proteins was observed between stages 2 and $3(p<0.05)$. Table 1 and Fig 1B show the serum levels of hs-CRP in the ASO groups; they were higher than in the non-ASO patients $(\mathrm{p}<0.0001)$ and increased as the disease developed $(\mathrm{p}<0.05)$.

Fig 2 shows a typical result of the analysis of $\mathrm{S}$ glutathionylated proteins using biotin-GST. SDS-PAGE profiles did not differ between sera from non-ASO patients and sera from ASO patients under reduced (Fig 2A, lanes 2, 3 ) or non-reduced (lanes 4, 5) conditions. S-glutathionylated protein bands were detected more in ASO patients than in non-ASO patients under non-reduced conditions (Fig 2B). In vitro, levels of S-glutathionylated proteins were reduced weakly in the presence of the GSH/GSSG system (Fig 2C, lane 2), and strongly in the presence of the GSH/GSSG system and GRX (lane 3). This suggests that the increase in the serum levels of S-glutathionylated proteins reflects a reduced redox regulation in ASO patients. Immunoprecipitation of proteins by anti-apolipoprotein B100 (apoB100) and treatment with biotin-GST revealed that apoB100 is Sglutathionylated in ASO (Fig 2D), which suggests that the S-glutathionylation of proteins in serum involves apoB100.

The serum levels of total cholesterol and LDL-C were higher in the non-ASO patients than in the ASO patients; however, there was no correlation between the levels of Sglutathionylated proteins and those of total cholesterol or LDL-C. Similarly, the levels of S-glutathionylated proteins did not relate to the levels of triglyceride in serum (data not shown).

\section{Relationship Between S-Glutathionylated Proteins and ABI}

The relationship between S-glutathionylated proteins and $\mathrm{ABI}$ was analyzed using a multiple linear regression model with covariates (Table 2). The coefficient, standard error, and p-value of S-glutathionylated proteins were $-0.0455,0.0173$, and 0.0105 , respectively. Similarly, the pvalue of both hypertension and smoking was less than 0.05 . The data suggest that formation of S-glutathionylated 
A

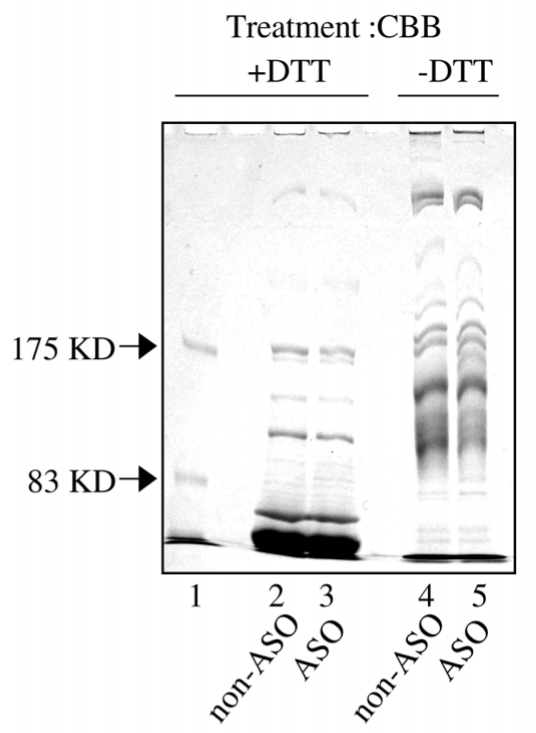

C

Treatment : Biotin-GST

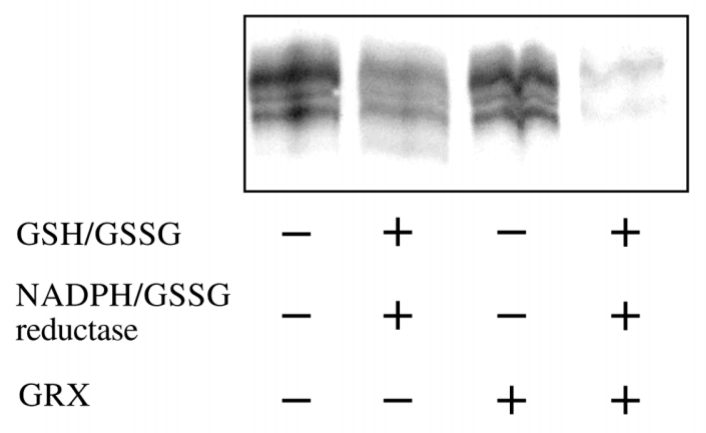

B

Treatment : Biotin-GST

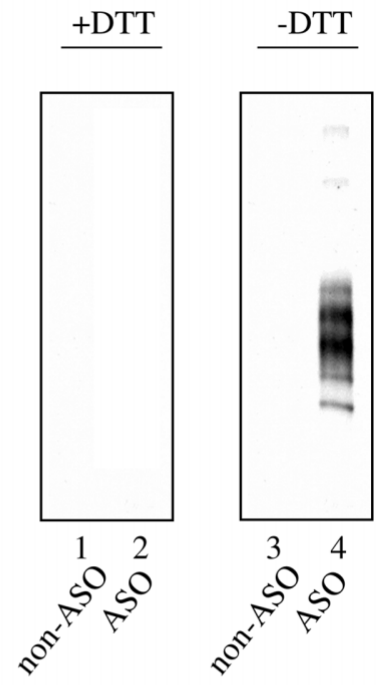

D

IP: Anti-apoB100
Treatment: Biotin-GST

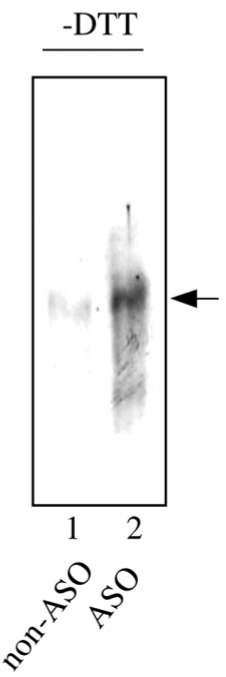

Fig 2. Characteristics of S-glutathionylated proteins. Serum samples from a patient with ASO and another with non-ASO was subjected to 5\% SDS-PAGE and analyzed using biotin-GST. (A) CBB stain. Lane 1, molecular weight standard; lane 2, nonASO with DTT; lane 3, patient with DTT; lane 4, non-ASO without DTT; lane 5, patient without DTT. (B) Staining using biotin-GST under reducing conditions (1 and 2) and non-reducing conditions (3 and 4). (C) Effect of the GSH/GRX system on the S-glutathionylated proteins. From the serum sample of a patient with ASO $1 \mu 1$ was treated in buffer containing components of the GSH/GRX system for $30 \mathrm{~min}$ at $30^{\circ} \mathrm{C} . \mathrm{GSH} / \mathrm{GSSG}, 1 \mathrm{mmol} / \mathrm{L} \mathrm{GSH}$ and $0.05 \mathrm{mmol} / \mathrm{L}$ GSSG; NADPH/GSSG reductase, $1 \mathrm{mmol} / \mathrm{L}$ NADPH and 1.2 units of GSSG reductase; $1 \mu \mathrm{g}$ of GRX (16 samples were subjected to 5\% SDS-PAGE under non-reducing conditions and analyzed using biotin-GST. (D) Serum was immunoprecipitated by anti-apoB100 antibody, subjected to $5 \%$ SDS-PAGE under non-reducing conditions, and analyzed using biotin-GST. Lane 1, non-ASO patient; lane 2, ASO patient. The arrow indicates a band corresponding to apoB100 protein. ApoB100, apolipoprotein B100; ASO, arteriosclerosis obliterans; CBB, coomassie brilliant-blue; DTT, dithiothreitol; GRX, glutaredoxin; GSH, glutathione; GST, glutathione Stransferase; GSSG, the reduced form of glutathione; SDS-PAGE, sodium dodecyl sulfate-polyacrylamide gels.

Table 2 Multiple Linear Regression Model for ABI

\begin{tabular}{lccl}
\hline \hline Variables & Coefficient & Standard error & $p$ value \\
\hline S-glutathionylated proteins (relative intensity) & -0.0455 & 0.0173 & 0.0105 \\
Hypertension* & -0.189 & 0.0622 & 0.00338 \\
Diabetes* & 0.0275 & 0.0503 & 0.587 \\
Hypercholesterolemia* & -0.0229 & 0.0514 & 0.657 \\
Smoking* & -0.170 & 0.0512 & 0.00142 \\
hs-CRP $(\mathrm{ng} / \mathrm{ml}) * *$ & -0.0513 & 0.0286 & 0.0774
\end{tabular}

*Positives are defined as 1. Negatives are defined as $0 . * *$ Values for hs-CRP were transformed in logarithm of 10.

Abbreviations see in Table 1.

proteins in serum is involved in the progress of ASO.

\section{Discussion}

As to the diagnosis of ASO, several tests, such as angiography, estimations of $\mathrm{ABI}$ and PWV, and measurements of circulating levels of hs-CRP, have been used to detect PAD. However, these estimations are not sufficient to predict the development of ASO in its earlier stages.

Oxidative stress is a principle cause of aging and the development of diseases such as inflammation, infection, cancer, and cardiovascular disorders9,10 Exogenous or endogenous sources of oxidative stress and weakened antioxidative defenses can damage macromolecules such as 
DNA, lipids, and proteins. The levels of molecules modified by oxidative stress can be estimated; however, there are currently no sensitive and specific methods to evaluate the oxidative stress-induced development of cardiovascular diseases.

The redox system regulates certain protein functions and protects cells from $\mathrm{H}_{2} \mathrm{O}_{2}$-induced apoptosis. ${ }^{16}$ TRX is a protein that is ubiquitously expressed in all living cells and which fulfils a variety of biological functions related to cell proliferation and apoptosis. ${ }^{17}$ Increases in serum TRX levels have been found in patients with various coronary risk factors, such as smoking, hypertension, and hypercholesterolemia. ${ }^{18}$ Increases in S-glutathionylated proteins have been found in ischemic preconditioned hearts 19 Those reports suggest that chronic oxidative stress may be involved in the progression of the coronary diseases associated with risk factors. As to the role of GSH/GRX, we previously found that the anti-apoptotic activity of Akt is regulated by the GSH/GRX system inside the cell, ${ }^{16}$ which led us to speculate that an imbalance of the redox state in serum reflects an impairment of circulatory compartments by oxidative stress, and we became interested in estimating the levels of S-glutathionylated proteins in serum as a marker for the risk of developing peripheral vascular damage. In the present study, serum levels of S-glutathionylated proteins were elevated in the earlier stages of ASO (Fig 1A). Levels of S-glutathionylated proteins in sera from ASO patients were reduced in the presence of the GSH/GRX system (Fig 2C). These results strongly suggest that during the development of ASO, chronic oxidative stress induces an imbalance of the redox state and protein thiols are oxidized in the serum of patients with ASO, although the mechanism of redox regulation to maintain the reduced form of cysteine thiols is not well understood. The application of anti-oxidant therapies, such as $\alpha$-tocopherol ${ }^{18}{ }^{18}$ statins ${ }^{20}$ or exercise ${ }^{21}$ may improve the redox imbalance and reduce the levels of S-glutathionylated proteins in ASO. If so, estimation of S-glutathionylated proteins is useful as a marker for the success of therapies and trials may be warranted.

Redox-active cysteine residues in the albumin of human serum have been reported;22 however, under the experimental conditions used in the present study, we could not identify S-glutathionylated albumin (data not shown). We found that apoB100 protein is S-glutathionylated (Fig 2D). At present, it is unclear if the thiol-modification of apoB 100 affects its function.

The method we used for the estimation of S-glutathionylated proteins used biotinylated GST. However, methods using electrophoresis are neither simple nor sensitive. Attempts have been made to detect $\mathrm{S}$-glutathionylated proteins by a proteomic approach using ${ }^{35} \mathrm{~S}$-labeled GSH in vitro, but this is not a convenient method ${ }^{2} 3$ Further development of a widely applicable method, such as enzyme-linked immunosorbent assay, is required for use with clinical samples.

ASO is an atherosclerotic peripheral occlusive disease. Oxidized LDL (ox-LDL) appears to play a key role in atherogenesis? ${ }^{24}$ A circular Ox-LDL, a product of oxidative stress, has been reported in patients with hyperlipidemia.25,26 In the present study, there was no relation between lipid metabolism and levels of S-glutathionylated proteins (data not shown), and a pathological comparison of S-glutathionylated proteins with ox-LDL was not conducted.

The relationship between various risk-markers and the development of ASO was analyzed with a multiple regression model (Table 2). The data suggested that levels of
S-glutathionylated proteins in serum are a risk-marker for ASO. Similarly, cigarette smoking was found to correlate with a decrease in ABI, which is consistent with a report that smoking induces low-grade inflammation and thrombogenicity ${ }^{27}$ as well as chronic obstructive pulmonary disease 28 It should be taken into account that there are many other factors influencing the progress of ASO, such as drugs, duration of accompanying diseases, genetic background, etc and the analysis here is not sufficient to rule out other risk factors; however, the increase in the serum levels of S-glutathionylated proteins may, at least in part, reflect progression of ASO.

In summary, the S-glutathionylation of proteins in serum may reflect the redox imbalance induced by oxidative stress and play a role in the development of ASO.

\section{Acknowledgments}

This work was supported in part by Grants-in-aid for Scientific Research from the Ministry of Health, Labor and Welfare of Japan, and Technology through the 21st Century CENTER OF EXCELLENCE Program, and by a research grant for health sciences from the Japanese Ministry of Health and Welfare.

We are grateful to Akiko Emura for excellent technical assistance.

\section{References}

1. Second European consensus document on chronic critical leg ischemia. Circulation 1991; 84(Suppl 4): IV-1 - IV-26.

2. Makino H, Aoki M, Hashiya N, Yamasaki K, Hiraoka K, Shimizu H, et al. Increase in peripheral blood flow by intravenous administration of prostaglandin E1 in patients with peripheral arterial disease, accompanied by up-regulation of hepatocyte growth factor. Hypertension Res 2004; 27: 85-91.

3. Morishita R, Aoki M, Hashiya N, Makino H, Yamasaki K, Azuma J, et al. Safety evaluation of clinical gene therapy using hepatocyte growth factor to treat peripheral arterial disease. Hypertension 2004; 44: $203-209$.

4. Berliner JA, Territo MC, Sevanian A, Ramin S, Kim JA, Bamshad B, et al. Minimally modified low density lipoprotein stimulates monocyte endothelial interactions. J Clin Invest 1990; 85: 1260-1266.

5. Malden LT, Chit A, Raimes FW, Ross R. The influence of oxidatively modified low density lipoprotein on expression of platelet-derived growth factor by human monocyte-derived macrophages. $\mathrm{J}$ Biol Chem 1991; 25: 213-222.

6. Gibbons GH, Dzau VJ. The emerging concept of vascular remodeling. N Engl J Med 1994; 30: 1431-1438.

7. Stadtman ER. Importance of individuality in oxidative stress and aging. Free Radic Biol Med 2002; 33: 597-604.

8. Cheng G, Ikeda Y, Iuchi Y, Fuji J. Detection of S-glutathionylated proteins by glutathione S-transferase overlay. Arch Biochem Biophys 2005; 435: 42-49.

9. Berlett BS, Stadtman ER. Protein oxidation in aging, disease, and oxidative stress. J Biol Chem 1997; 272: 20313-20316.

10. Finkel T, Holbrook NJ. Oxidants, oxidative stress and the biology of aging. Nature 2000; 408: 239-247.

11. Padgett CM, Whorton AR. Cellular responses to nitric oxide: Role of protein S-thiolation/dethiolation. Arch Biochem Biophys 1998; 358: 232-242.

12. Mohr SJ, Hallk H, de Biotte A, Lapetina EG, Brune B. Nitric oxideinduced S-glutathionylation and inactivation of glyceraldehydes3-phosphate dehydrogenase. J Biol Chem 1999; 274: $9427-9430$.

13. Caplan JF, Filipenko NR, Fitzpatrick SL, Waisman DM. Regulation of annexin A2 by reversible glutathionylation. Biol Chem 2004; 279: $7740-7750$.

14. Ward NE, Pierce DS, Chungt SE, Gravitt KR, O'Brian CA. Irreversible inactivation of protein kinase $\mathrm{C}$ by glutathione. J Biol Chem 1998; 273: $12558-12566$.

15. Cabiscol E, Levine RL. The phosphatase activity of carbonic anhydrase III is reversibly regulated by glutathiolation. Proc Natl Acad Sci USA 1996; 93: 4170-4174.

16. Murata H, Ihara Y, Nakamura H, Yodoi J, Sumikawa K, Kondo T. Glutaredin exerts antiapoptotic effect by regulating the redox state of Akt. Biol Chem 2003; 278: 50226-50233.

17. Yamawaki H, Haendeler J, Berk BC, Thioredoxin: A key regulator of cardiovascular homeostasis. Circ Res 2003; 93: 1029-1033. 
18. Miwa K, Kishimoto C, Nakamura H, Makita T, Ishii K, Okuda N, et al. Serum thioredoxin and a-tocopherol concentrations in patients with major risk factors. Circ J 2005; 69: 291 - 294.

19. Eaton P, Bell RM, Cave AC, Shattock MJ. Ischemic predonditioning: A potential role for protein S-thiolation? Antioxid Redox Signal 2005; 7-8: 882-888.

20. Yokoi H, Nobuyoshi M, Mitsudo K, Kawaguchi A, Yamamoto A, ATHEROMA Study Investigators. Three-year follow-up results of angiographic intervention trial using an HMG-CoA reductase inhibitor to evaluate retardation of obstructive multiple atheroma (ATHEROMA) Study. Circ J 2005; 69: 875-883.

21. Maejima Y, Ysu T, Ueba H, Kobayashi N, Hashimoto S, Kubo N, et al. Exercise after heparin administration: New therapeutic program for patients with non-option arteriosclerosis obliterans. Circ J 2005; 69: $1099-1104$

22. Cha MK, Kim IH. Disulfide between Cys392 and Cys438 of human serum albumin is redox-active, which is responsible for the thiorexin-supported lipid peroxidase activity. Arch Biochem Biophys 2006; 445: 19-25.

23. Jung $\mathrm{CH}$, Thomas JA. S-glutathionylated hepatocyte proteins and insulin disulfides as substrates for reduction by glutaredoxin, thiore- doxin, protein disulfide isomerase, and glutathione. Arch Biochem Biophys 1996; 335: 61-72.

24. Mukai E, Kume N, Hayashida K, Minami M, Yamada Y, Seino Y, et al. Heparin-bindin EGF-like growth factor induces expression of lectin-like oxidized LDL-receptor-1 in vascular smooth muscle cells. Atherosclerosis 2004; 176: 289-296.

25. Barros MR, Bertolami MC, Aballa DS, Ferreira EP. Identification of mildly oxidized low-density lipoprotein (electronegative LDL) and its auto-antibodies $\mathrm{IgG}$ in children and adolescents hypercholesterolemic offspring. Atherosclerosis 2006; 184: 103-107.

26. Zhang B, Noda K, Matsunaga A, Kumagai K, Saku K. A comparative crossover study of the effects of fluvastatin and pravastatin (EPCOS) on circulating autoantibodies to oxidized LDL in patients with hypercholesterolemia. J Atheroscler Thromb 2005; 12: 41-47.

27. Yasue H, Hirai N, Mizuno Y, Harada E, Itoh T, Yoshimura M, et al. Low-grade inflammation, thrombogenicity, and atherogenic lipid profile in cigarette smokers. Circ J 2006; 70: 8-13.

28. Nagai K, Betsuyaku T, Kondo T, Nasuhara Y, Nishimura M. Long term smoking with age builds up excessive oxidative stress in bronchoalveolar lavage fluid. Thorax 2006; 61: 496-502. 